\title{
Beta 3 subunit of G-protein and its influence on autonomic nervous system in patients with vasovagal syncope.
}

\author{
Evin $\mathrm{L}^{1}$, Mitro $\mathrm{P}^{1}$, Habalova $\mathrm{V}^{2}$, Simurda $\mathrm{M}^{1}$, Muller $\mathrm{E}^{1}$, Murin $\mathrm{P}^{1}$ \\ Department of Cardiology, VUSCH, Kosice, Slovakia. evin.lukas@gmail.com
}

\begin{abstract}
OBJECTIVES: The aim of this prospective study was to investigate the impact of genetic polymorphisms of $\beta 3$ subunit of G-protein on the occurrence of vasovagal syncope, hemodynamic parameters and heart rate variability during head-up tilt test (HUT).

BACKGROUND: G-proteins play an important role in the intracellular transmission of impulses in cardiovascular autonomic reflexes.

METHODS: In 157 patients with suspected vasovagal syncope HUT was performed. Ninety-one patients (38 men, 53 women, mean age $48 \pm 17$ years) had positive HUT. Control group consisted of 109 subjects (69 men, 40 women, mean age $37 \pm 16$ years) with no history of syncope. Results of HUT, hemodynamic parameters and LF, HF, LF/HF, SDNN, RMSSD parameters of heart rate variability were compared in patients with different genotypes. C825T polymorphism of $\beta 3$ subunit of G-protein was determined in the study subjects.

RESULTS: There was no significant difference in the distribution of genotypes between patients and control group. Also, there was no significant difference in hemodynamic parameters. A statistically significant difference was found between genotypes in LF/HF in the early HUT (mean rank CC: 48.68 vs CT: 35.51 vs TT: $34.14 ; p$ $=0.039$ ) and at RMSSD at the time of syncope (mean rank CC: 32.38 vs CT: 42.74 vs TT: $18.50 ; p=0.026$ ). CONCLUSIONS: In this study, the relation of C825T polymorphism of $\beta 3$ subunit of G-protein to vasovagal syncope was not documented (Tab. 2, Fig. 4, Ref. 37). Text in PDF www.elis.sk.

KEY WORDS: vasovagal syncope, HUT test, heart rate variability, gene polymorphisms, $\beta 3$ subunit of G-protein.
\end{abstract}

\section{Introduction}

Vasovagal syncope (VVS) is the most common cause of transient loss of consciousness. Most people have one or few episodes during life, while others suffer from frequent episodes (1). According to Sheldon et al the most common age of onset of the first syncope is 13 years (2).

The exact pathogenetic mechanism is not yet fully understood. It is known that the predisposition of the neurovascular syncope may occur more frequently in some families $(3,4,5,6)$. These observations suggest some association between VVS and heredity.

In relation to this matter some gene polymorphisms were investigated. In particular, polymorphisms of the renin-angiotensin system, and serotonin and endothelin system the genes encoding the $\alpha$ la adrenergic receptor, the genes encoding G-protein and the sympathetic nervous system were studied $(7,8,9,10,11,12$, $13,14,15)$.

${ }^{1}$ Department of Cardiology, VUSCH, Kosice, Slovakia, and ${ }^{2}$ Department of Medical Biology, LF UPJŠ, Kosice, Slovakia

Address for correspondence: L. Evin, MD, Department of Cardiology, VUSCH, Ondavska 8, SK-040 11 Kosice, Slovakia.

Phone: +421.904412670 ,

Acknowledgements: The project was in the framework of the VEGA grant 1/0479/10: Gene polymorphisms in patients with vasovagal syncope.
The molecular mechanism of intracellular signals transduction plays an important role in cardiovascular reflexes. Heterotrimeric G-protein is crucial for signal transduction in cardiovascular reflexes, via G-protein coupled receptor responses (16). Models for activation of G-coupled receptors suppose that these receptors are in equilibrium between the activated and non-activated state. $\mathrm{G}$ proteins consist of three subunits, also referred to as $\alpha$, $\beta, \gamma . \alpha$-subunit contains a guanine nucleotide binding site, and in inactivated state guanosine diphosphate (GDP) is bound to the subunit. Activation of G-protein is mediated by the binding of agonist, e.g. catecholamines, to the adrenergic receptor. During this activation there is a conformational change and the exchange of GDP bound to the $\alpha$-subunit of G for guanosine triphosphate (GTP). Once binding occurs, GTP, the G- $\alpha$ GTP complex dissociates from the $\beta \gamma$-subunits of $\mathrm{G}$, whereas the two subunits remain tightly bound in the membrane. After hydrolysis of the GTP, inactivated G- $\alpha$ subunit re-associates rapidly with the G- $\beta \gamma$ subunit. After activation, the dissociated subunits, both G- $\beta \gamma$ and G- $\alpha$ GTP, are able to activate various signaling cascades, and effectors proteins. The effector molecules of $\mathrm{G} \beta \gamma$ heterodimer are specific subtypes of phospholipase $C, \beta$-adrenergic receptor kinases, and $\mathrm{K}+$ and $\mathrm{Ca}^{2+}$-specific ion channels (17). There are five genes that encode $\beta$-subunit of G-protein (16). The gene responsible for the $\beta 3$ subunit of G-protein (GNB3) is located on chromosome 12p (18). A single nucleotide polymorphism (SNP - Single Nucleo- 
tide Polymorphism), C825T is located on exon 10, leading to the exchange of a cytosine for thymidine at position 825 in the cDNA (19). That causes the intracellular signal transfer and increased vascular reactivity (20).

C825T polymorphism of $\beta 3$ subunit of G-protein has been observed in several studies. $825 \mathrm{~T}$ allele was observed in association with left ventricular hypertrophy, obesity, insulin resistance $(21,22,23)$.

GNB3 was also studied in relation to cardiovascular processes. In Luo et al meta-analysis of 36802 subjects, association of a this polymorphism with risk of essential hypertension was observed (24). Nakao et al investigated this polymorphism in relation to postural orthostatic tachycardia syndrome (28). In connection with VVS 4 paperswere published so far. However, their results were conflicting $(11,12,13,15)$.

We can presume that VVS may be associated with this molecular mechanism. The aim of this study was to investigate the relation of genetic polymorphism of $\beta 3$ subunit of G-protein related to blood pressure (BP), heart rate and heart rate variability (HRV) during the head-up tilt table test (HUT test).

\section{Methods}

Informed consent was obtained from all participants in the study. This was a prospective study. It consisted of 157 patients with history of at least one syncope. Following the standard diagnostic algorithm, patients were subjected to HUT test and divided into groups. Patient group (group A) consisted of 91 subjects with a positive HUT test, 53 women with mean age $47.50 \pm 17.49$ years and 38 men with mean age $49.77 \pm 16.95$ years. The control group consisted of 109 randomly selected individuals with no history of syncope and pre-syncope (group B). These were 40 women, with mean age $36.85 \pm 16.37$ years and 69 men with mean age 38.51 \pm 16.22 years APart of the control group was willing to undergo HUT test (42 subjects). HUT test was negative in 18 subjects (group C) out of whom 6 were women with mean age $23 \pm 2.36$ years and 12 were men with mean age $23.41 \pm 2.74$ years (Fig. 1).

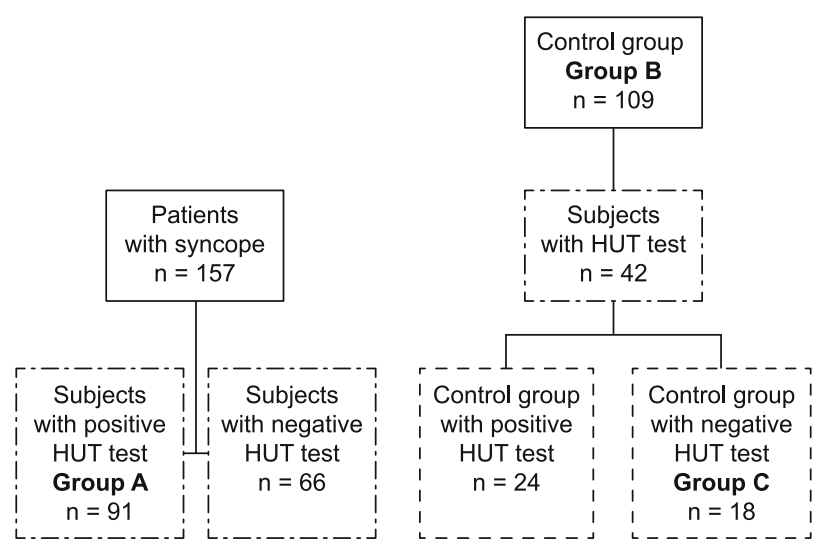

Fig. 1. Organization scheme of selection of patients with VVS and control groups.
HUT was carried out according to the Italian protocol before midday in room with constant temperature. Before the test, $10 \mathrm{ml}$ of patient's peripheral blood was drawn. Following a 10 minute stabilization of hemodynamics in the horizontal position, subjects were tilted to a position of $70^{\circ}$ for 20 minutes. If no syncope occurred during this period of time, the test continued to an active phase lasting for 15 minutes, where patients received $400 \mu \mathrm{g}$ of nitroglycerine by sublingual spray application. The HUT test was completed by tilting back in a horizontal position in the event of syncope or accentuated pre-syncope with decrease in systolic blood pressure (SBP) under $80 \mathrm{mmHg}$ and /or bradycardia below $50 /$ minute. The test was considered positive when spontaneous symptoms of syncope or pre-syncope were reproduced during HUT test and confirmed by patient.

Blood pressure and ECG were continuously recorded through Finapress Medical System ${ }^{\mathrm{TM}}$ and evaluated by the use of BeatsScope Easy ${ }^{\mathrm{TM}}$. Heart rate variability (HRV) has been compiled with the use of Kubios HRV TM software in following five minute intervals: basal phase ( 5 minutes before tilting), early tilt phase ( 5 minutes after tilting), end of the passive phase (15-20 minutes after tilting), syncope phase ( 5 minutes before syncope) and recovery phase (5 minutes after tilting/end test). LF, HF, LF/HF, SDNN, and RMSSD parameters of HRV were compared in patients with positive HUT test (Group A) and the control group with a negative HUT test (Group C).

\section{Genotyping}

Genotyping was performed from peripheral blood taken from the patient before the HUT test (Group A), and also from control group in lying position at rest (Group B). DNA was extracted from lymphocytes in the blood. All samples were immediately centrifuged, frozen and stored at $-80^{\circ} \mathrm{C}$ until their assay.

Standard polymerase chain reaction was used to determine genetic polymorphisms. C825T polymorphism of $\beta 3$ subunit of G-protein was the subject of current analysis.

\section{Statistical analysis}

Variables with normal distribution of data were analyzed by Students' t-test, analysis of variance (ANOVA) and presented as averages with standard deviations. Variables outside the normal distribution were evaluated by the Mann-Whitney U-test and KruskalWallis $\mathrm{H}$ test and presented as a mean rank plus average and standard deviations. Parameters of HRV were out of normal distribution, even after use of logarithmic transformation. Categorical variables were presented as numbers or percentages and analyzed with the help of the Pearson $\chi^{2}$ test. $p$ value $<0.05$ was considered statistically significant. SPSS ${ }^{\text {TM }}\left(18^{\text {th }}\right.$ version) was used to analyze variables.

\section{Results}

Distribution of genotypes in patients with syncope (group A) was: CC $47.25 \%$ (43), CT $46.15 \%$ (42), TT $6.59 \%$ (6), see graph n. 1. Distribution of genotypes in the control group (Group B) was: CC $52.29 \%$ (57), CT $40.36 \%$ (44) TT $7.34 \%$ (8) (Fig. 2). Syncope group and control group were at Hardy-Weinberg equilibrium. No 
Patients with syncope

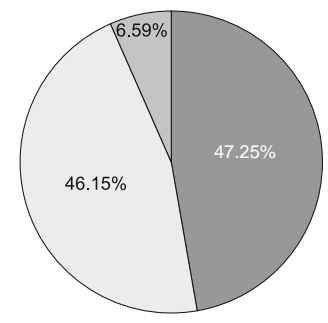

$\square \mathrm{CC}$
Control group

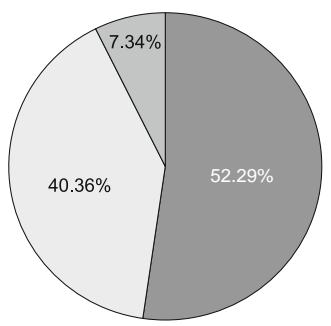

TT
Fig. 2. Distribution of specific genotypes in patient and control group.

statistically significant difference was observed $(p=n s)$ between patients with syncope and the control group with respect to the presence of various genotypes. Distribution by VVS types was classified according to VASIS classification and reported as follows: mixed type (I) $42.86 \%$ (39), cardioinhibitory type (II) $9.89 \%$ (9), vasodepressor type (III) $47.25 \%$ (43). Distribution of VVS types according to specific genotypes was also made. In patient group with CC genotype there were $34.21 \%$ (13) of mixed type; $10.53 \%$ (4) of cardioinhibitory; $55.26 \%$ (21) of vasodepressor; for the CT genotype group there were $51.16 \%$ (22) mixed type; $9.3 \%$ (4) of cardioinhibitory type; $39.53 \%$ (17) of vasodepressor type; for the TT genotype group: $40 \%$ (4) of mixed; $10 \%$ (1) of cardioinhibitory and $50 \%$ (5) of vasodepressor type. There was no significant difference observed between the VVS types in patients with various genotypes.

Also, there was no statistically significant difference in blood pressure and heart rate values during HUT in different genotypes (Tab. 1).

LF and HF did not differ during the test among CC, CT and TT genotypes. LF/HF was significantly different in the early tilt phase (mean rank CC: 48.68 vs CT: 35.51 vs TT: $34.14 ; \mathrm{p}=0.039$ ).
When assessing each genotype separately, significant difference between CT and CC genotype ( $\mathrm{p}=0.002)$ was observed.

SDNN during the HUT test was without significant difference between groups. RMSSD parameter at the time of syncope has reached significant difference (mean rank CC: 32.38 vs CT: 42.74 vs TT: $18.50 ; p=0.026)$. In a subsequent analysis of genotypes, we have observed a significant difference between CC and CT type ( $\mathrm{p}$ $=0.036)$ and borderline difference between the CT and TT genotype $(p=0.052)$. Values of heart rate variability and its changes during the HUT test are summarized in Table 2 and Figs 3 and 4.

\section{Discussion}

G-proteins are part of several pathophysiological processes, through their G protein-coupled receptors. On account of G-protein, signals are transmitted from the extracellular space into the cell and further through the second and third messengers. Adrenergic and muscarinic receptors, main receptors of the sympathetic and the parasympathetic system, belong to the family of receptors coupled with G-proteins.

Therefore we can assume certain modulation of the autonomic nervous system with a change of these proteins. Siffert et al first observed that the $\mathrm{T}$ allele of $825 \mathrm{C} / \mathrm{T}$ polymorphism of $\beta 3$ subunit of G-protein is associated with an increased intracellular signal transduction. In the pathophysiology of vasovagal syncope we presume a central role of the autonomous nervous system and various changes at the level of the sympathetic and parasympathetic systems in particular (20).

Lelonek et al first suggested a possible association of C825T polymorphisms of G-protein in patients with VVS (11). They observed CC genotype more frequently in syncope group. Another work from the same group found a statistically significant association of TT genotype with the absence of syncope during HUT test in 217 patients (12).

In another study, this group of authors besides 307 vasovagal patients included also the control group which consisted of 74

Tab. 1. Changes of hemodynamic parameters during HUT test.

\begin{tabular}{|c|c|c|c|c|}
\hline \multirow{2}{*}{ Phases of HUT test } & \multicolumn{3}{|c|}{ GNB3 genotypes } & \multirow{2}{*}{$\mathrm{P}$} \\
\hline & $\mathrm{CC}$ & $\mathrm{CT}$ & $\mathrm{TT}$ & \\
\hline Supine SBP & $128.95 \pm 16.497$ & $135.09 \pm 19.505$ & $128.38 \pm 11.698$ & 0.232 \\
\hline Supine DBP & $77.43 \pm 10.894$ & $79.73 \pm 10.351$ & $81.00 \pm 9.695$ & 0.493 \\
\hline SBP 5 min & $127.89 \pm 23.134$ & $130.61 \pm 24.799$ & $125.25 \pm 19.939$ & 0.844 \\
\hline DBP 5 min & $78.06 \pm 13.915$ & $75.08 \pm 15.165$ & $74.00 \pm 8.718$ & 0.663 \\
\hline SBP during syncope & $78.65 \pm 22.770$ & $78.35 \pm 24.360$ & $79.14 \pm 20.643$ & 0.996 \\
\hline DBP during syncope & $53.05 \pm 22.323$ & $49.16 \pm 24.901$ & $40.57 \pm 31.015$ & 0.414 \\
\hline Difference in DBP $0-5 \mathrm{~min}$ & $-2.43 \pm 17.025$ & $3.75 \pm 12.634$ & $3.00 \pm 14.024$ & 0.216 \\
\hline Difference in basal and syncope SBP & $50.41 \pm 26.725$ & $56.83 \pm 29.270$ & $49.71 \pm 26.088$ & 0.548 \\
\hline Difference in basal and syncope DBP & $21.73 \pm 21.005$ & $29.37 \pm 23.377$ & $39.14 \pm 35.974$ & 0.120 \\
\hline $\mathrm{SBP}$ in recovery phase & $124.00 \pm 15.904$ & $124.61 \pm 20.490$ & $115.75 \pm 7.932$ & 0.649 \\
\hline DBP in recovery phase & $75.51 \pm 9.963$ & $75.72 \pm 11.724$ & $68.75 \pm 4.856$ & 0.461 \\
\hline Supine HR & $72.52 \pm 12.136$ & $70.16 \pm 8.353$ & $68.38 \pm 8.400$ & 0.415 \\
\hline HR $5 \mathrm{~min}$ & $84.93 \pm 14.340$ & $81.61 \pm 11.314$ & $78.88 \pm 18.917$ & 0.351 \\
\hline HR in recovery & $70.43 \pm 14.261$ & $68.14 \pm 11.559$ & $69.00 \pm 8.907$ & 0.754 \\
\hline
\end{tabular}

SBP - systolic blood pressure, DBP - diastolic blood pressure, HR - heart rate, NTG - nitroglycerin 
Tab. 2. Heart rate variability during HUT test.

\begin{tabular}{|c|c|c|c|c|c|c|c|}
\hline \multirow{3}{*}{ Phases of HUT test } & \multicolumn{6}{|c|}{ GNB3 genotypes } & \multirow{3}{*}{$\mathrm{P}$} \\
\hline & \multicolumn{2}{|l|}{$\mathrm{CC}$} & \multicolumn{2}{|c|}{$\mathrm{CT}$} & \multicolumn{2}{|l|}{ TT } & \\
\hline & Average \pm SD & Mean Rank & Average \pm SD & Mean Rank & Average $\pm \mathrm{SD}$ & Mean Rank & \\
\hline LF supine basal & $11977.9 \pm 37098.5$ & 37.85 & $30781.5 \pm 155360.8$ & 37.33 & $1111.7 \pm 1009.9$ & 31.43 & 0.762 \\
\hline LF $0-5 \mathrm{~min}$ & $10559.7 \pm 51103.2$ & 46.53 & $29715.9 \pm 102359.5$ & 37.49 & $909.8 \pm 1106.4$ & 35.43 & 0.202 \\
\hline $\mathrm{LF} 15-20 \mathrm{~min}$ & $2386.2 \pm 6107.8$ & 41.41 & $1364.2 \pm 1698$ & 39.78 & $1050.4 \pm 918.2$ & 39.43 & 0.948 \\
\hline LF syncope/25 min. & $1894.5 \pm 6142.2$ & 44.36 & $3500.5 \pm 11957.5$ & 42.41 & $612.1 \pm 717$ & 38.71 & 0.839 \\
\hline LF recovery & $4652.6 \pm 13749.2$ & 44 & $19073.9 \pm 89455.5$ & 42.18 & $1135.7 \pm 1628.5$ & 27.83 & 0.31 \\
\hline HF supine & $3691.2 \pm 21075.4$ & 36.33 & $5403.4 \pm 14054.1$ & 37.88 & $704.7 \pm 671.6$ & 36 & 0.949 \\
\hline $\mathrm{HF} 0-5 \mathrm{~min}$ & $4813.6 \pm 19610.7$ & 42.26 & $12180.4 \pm 42793.6$ & 40.89 & $308.4 \pm 283.1$ & 40.57 & 0.964 \\
\hline $\mathrm{HF} 15-20 \mathrm{~min}$ & $712.9 \pm 2571.1$ & 38.08 & $1347.6 \pm 4123.6$ & 42.64 & $4865.9 \pm 12184.1$ & 42.29 & 0.688 \\
\hline HF syncope/25 min & $991 \pm 3059.6$ & 41.31 & $1999.5 \pm 5984.6$ & 45.28 & $235.4 \pm 235.5$ & 39.71 & 0.726 \\
\hline HF recovery & $3828.2 \pm 10857.2$ & 42.33 & $8649.5 \pm 29844.7$ & 43.63 & $728.4 \pm 982.7$ & 29.5 & 0.408 \\
\hline LF/HF supine & $5.5 \pm 13$ & 41.06 & $3.1 \pm 3.7$ & 35 & $1.5 \pm 0.6$ & 27.29 & 0.227 \\
\hline $\mathrm{LF} / \mathrm{HF} 0-5 \mathrm{~min}$ & $10.3 \pm 32.4$ & 48.68 & $3.2 \pm 3.3$ & 35.51 & $2.6 \pm 0.8$ & 34.14 & 0.039 \\
\hline LF/HF 15-20 min. & $5.2 \pm 4.6$ & 44.89 & $3.3 \pm 2.9$ & 34.97 & $5.5 \pm 5.2$ & 45.71 & 0.156 \\
\hline LF/HF recovery & $3.2 \pm 4.2$ & 44 & $2.6 \pm 2.9$ & 40.34 & $1.9 \pm 1$ & 39.5 & 0.774 \\
\hline SDNN supine & $102.2 \pm 151.7$ & 38.58 & $91.5 \pm 76.2$ & 40.49 & $80 \pm 70.8$ & 33.94 & 0.77 \\
\hline SDNN 0-5 min & $68.9 \pm 52.8$ & 45.6 & $65.7 \pm 50.2$ & 40.26 & $52.7 \pm 22.2$ & 37.36 & 0.532 \\
\hline SDNN 15-20 min & $1808.8 \pm 10652.7$ & 39.92 & $60.8 \pm 39.9$ & 41.1 & $53.7 \pm 20.5$ & 40.5 & 0.977 \\
\hline SDNN syncope/25 min & $85.5 \pm 57.9$ & 39.28 & $95 \pm 45$ & 48.18 & $79.9 \pm 50.5$ & 34.86 & 0.186 \\
\hline SDNN recovery & $121.8 \pm 67.5$ & 45.55 & $129.9 \pm 154.6$ & 39.89 & $82.6 \pm 33.4$ & 32.25 & 0.347 \\
\hline RMSSD supine & $77.3 \pm 101.1$ & 29.65 & $94 \pm 102.4$ & 35.38 & $113.8 \pm 143.3$ & 33.75 & 0.487 \\
\hline RMSSD 0-5 min & $54.6 \pm 87$ & 35.12 & $1215.7 \pm 6598.8$ & 37.61 & $31.2 \pm 22.1$ & 30.25 & 0.751 \\
\hline RMSSD 15-20 min & $37.6 \pm 52.5$ & 31.34 & $54.7 \pm 64.5$ & 27.55 & $26.2 \pm 11.4$ & 27.75 & 0.362 \\
\hline RMSSD syncope/25 min & $43.7 \pm 57.1$ & 32.38 & $706.9 \pm 3730.4$ & 42.74 & $21 \pm 9.6$ & 18.5 & 0.026 \\
\hline RMSSD recovery & $86.3 \pm 96.1$ & 34.74 & $115.9 \pm 162.1$ & 36.88 & $57.4 \pm 37.3$ & 29 & 0.777 \\
\hline
\end{tabular}

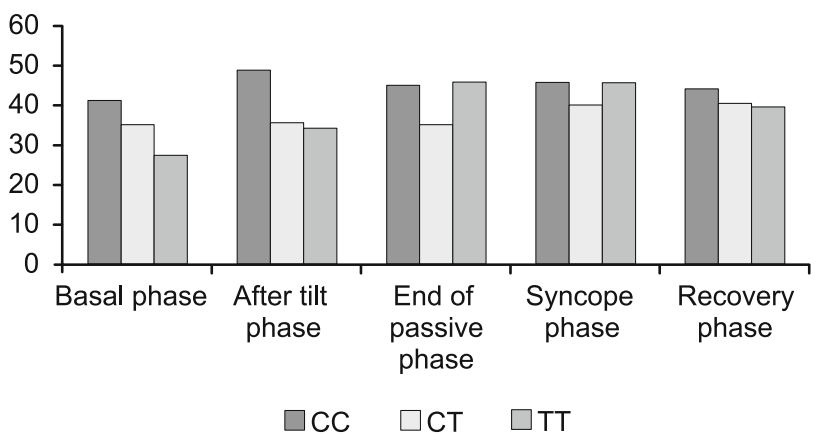

Fig. 3. Changes in LF/HF during HUT test. *There was statistically significant difference between followed genotypes in the after tilt phase $(p=0.039)$.

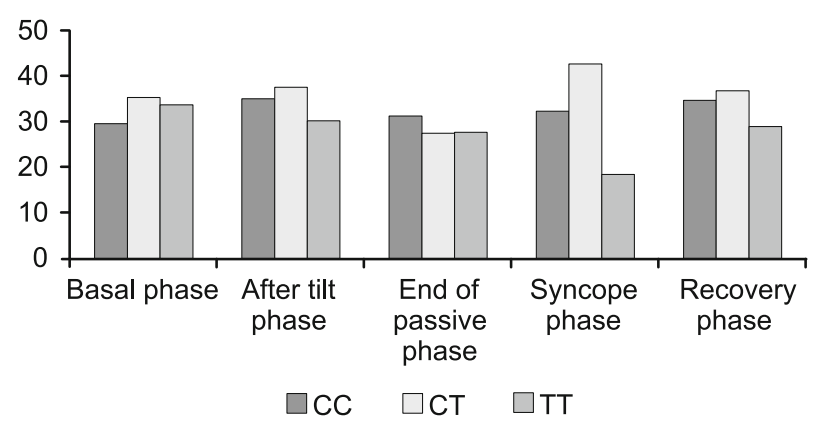

Fig. 4. Changes in RMSSD during HUT test. *There was statistically significant difference between followed genotypes in syncope phase $(p=0.026)$. healthy subjects with no history of syncope. C825T GNB3 polymorphisms were not associated with an increased incidence of malignant vasovagal syncope (13).

Sorrentino and co-workers tested 4 candidate genes polymorphisms, together with 825C/T GNB3, in relation to the VVS. 129 patients with syncope two months prior to enrollment underwent HUT test. Afterwards, these patients were divided according to their responses to HUT test. In conclusion, the authors did not observe any association between followed polymorphisms and results of HUT test (15).

In our research, there was no statistically significant difference in the proportions of genotypes between patients with vasovagal syncope and control group.

The above mentioned studies differ mainly in the control group. The first work had no control group (11). In two works control group consisted of patients with a history of syncope, but with negative HUT test $(12,15)$. Apart from our work, there was only one paper that had a control group of healthy individuals with no history of syncope (13). HUT test according to the Italian protocol has a sensitivity of $67.5 \%$ and a specificity of $85.7 \%$ (26). Due to that fact, division of individuals into patients and control group by positivity and negativity of HUT test may be misleading. In both groups (HUT test positive and negative) there might be patients with vasovagal etiology of syncope.

Present study is to our best knowledge the first published paper that described changes in heart rate variability during a HUT test with regards to C $825 \mathrm{~T}$ polymorphism of $\beta 3$ subunit of G-protein. 
142-147

In our work we observed a significant difference in RMSSD parameter in syncope phase between CC and CT type $(p=0.036)$ and borderline difference between the CT and TT genotype ( $\mathrm{p}=$ 0.052). It can be assumed that in patients with CT genotype there is a predominance of parasympathetic influence at the time of syncope. In our work there was also a significant difference observed in the LF/HF in early tilt phase after tilting. Patient with CC genotype had a higher LF/HF ratio compared to a CT genotype $(p=0.002)$. There was no difference between genotypes in $\mathrm{LF} / \mathrm{HF}$ value in basal phase. Comparing genotypes during basal phase up to tilting we found an increase in all parameters except for $\mathrm{CT}$. In patients with $\mathrm{CT}$ genotype, $\mathrm{LF} / \mathrm{HF}$ after tilting remained almost unchanged. Changes of HRV in CC genotype patients, in our study seem to be similar with changes described by Guzman et al for vasodepressor syncope (27). Vasodepressor type of syncope $(55.26 \%)$ was mainly represented in this genotype in our study. However, unlike Guzman's vasodepressor group of patients - we have described an increase in LF/HF parameter after tilting, which could be explained by the presence of cardioinhibitory and mixed type of syncope in this group of patients.

Different results have been described in various studies that evaluated HRV changes as a response to HUT test. Decrease in $\mathrm{HF}$ and increase in LF and LF/HF was often observed in studies that monitored changes in HRV during early stage of HUT test $(28,29,30,31)$. This could be interpreted as a decrease of parasympathetic and increase of sympathetic response to tilting from the horizontal position.

Contradictory results were reported in other studies. Kochiadakis et al described a decline in LF parameters in a positive HUT and no change in LF parameter in HUT negative patients (32). Kouakam et al observed a significant decrease in LF/HF after tilting during the first five minutes of test in HUT test positive patients, in contrast to HUT test negative patients. Authors of this study even postulated that this decline of LF/HF ratio during the first five minutes can be a predictor of HUT test induced syncope with $89 \%$ sensitivity and $89 \%$ specificity (33).

Stewart et al observed that LF and LF/HF parameters at the time of syncope were lower in patients with positive HUT tests in comparison to patients with negative HUT test. At the same time HF, SDNN, RMSSD parameters were higher in this group of patients (34). Evrengul with a team described a significant decrease in $\mathrm{LF}$ and LF/HF and a significant increase in HF parameters during syncope induced by HUT test (35). Furlan et al have described two distinct patterns of response in the autonomous vasovagal reaction. The first one was characterized by increased sympathetic modulation before syncope and second was characterized by progressive inhibition of sympathetic response until the syncope occurred. However, this observation was made in a group with no history of syncope (36). Hrubon et al described the changes of HRV in VVS patients according to the type of vasovagal syncope. During the vasodepressor syncope the spectral curve flattened almost to isoelectric line. Moreover, HF and LF parameters have almost disappeared. But when the patient was placed to the horizontal position there was a reflex activation of HF component. For cardioinhibitory syncope, the spectral output curve before syncope was at low amplitudes and increases together with LF and VLF. For syncope of mixed type, there was no typical spectral output (37).

In the studies described above, results of HRV during HUT test differ. Papers vary in the number of patients and the presence of the control group. Several works describe the various trends of HRV changes in different types of vasovagal syncope during HUT test, which should be considered when evaluating the results of heart rate variability. There is no uniform format of HRV changes during HUT test in patients with VVS that would be widely recognized.

\section{Limitations of the study}

The main limitation of this study is the number of participating subjects - especially the control group with no history of syncope was problematic as only a minor part was willing to undergo the HUT test.

Tracking of HRV changes in individual genotypes according to the type of VVS was complicated due to low cardioinhibitory syncope representation in both overall and in individual genotypes. This type of syncope was represented by less than five cases in each genotype in our population.

\section{Conclusions}

The study population did not show relationship of C825T polymorphism of $\beta 3$ subunit of G-protein in connection with vasovagal syncope.

\section{References}

1. Alboni P, Brignole M, Menozzi A et al. Clinical spectrum of neurally mediated reflex syncopes. Europace 2004; 5: 55-62.

2. Sheldon RS, Sheldon AG, Connolly SJ et al. Age of first faint in patients with vasovagal syncope. J Cardiovasc Electrophysiol 2006; 17: $49-54$.

3. Camfield PR, Camfield C. Syncope in childhood: a case control clinical study of the family tendency to faint. Can J Neurol Sci 17: 306-308.

4. Newton JL, Kenny R, Lawson J, Frearson R, Donaldson P. Prevalence of familial history in vasovagal syncope and haemodynamic response to head up tilt in first degree relatives. Clin Auton Res 13: 22-26.

5. Newton JL, Kerr S, Pairman J et al. Familial neurocardiogenic (vasovagal) syncope. Am J Med Genet A 133: 176-179.

6. Marquez MF, Urias KI, Hermosillo AG et al. Familial vasovagal syncope. Europace 7, 2005: 472-474.

7. Newton JL, Donaldson P, Parry S et al. Angiotensin converting enzyme insertion/deletion polymorphism in vasovagal syncope. Europace 7: 396-399.

8. Mudraková K, Mitro P, Salangovic J, Habalova V, Kirsch P, Tkac J. Gene polymorphisms of renin angiotensin system and serotonin transporter gene in patients with vasovagal syncope. Bratisl Lek Listy 2009;110 (2): 73-76.

9. Sorrentino S, Forleo C, Iacoviello M et al. Endothelin system polymorphisms in tilt test-induced vasovagal syncope. Clin Auton Res 2009; 19 (6): $347-354$. 
10. Hernández-Pacheco G, González-Hermosillo A, Murata C et al. Arg347Cys polymorphism of $\alpha 1$ a-adrenergic receptor in vasovagal syncope. Case-control study in a Mexican population. Auton Neurosci Basic Clin 2014; 183: 66-71.

11. Lelonek M, Pietrucha T, Stanczyk A, Goch JH. Vasovagal syncope and the C825T GNB3 polymorphism. Anatol J Cardiol 2007: 7 (Suppl 1): 206-208.

12. Lelonek M, Pietrucha T, Matyjaszczyk M, Goch JH. A novel approach to syncopal patients: association analysis of polymorphisms in Gprotein genes and tilt outcome. Europace 11: 89-93.

13. Lelonek M, Pietrucha T, Matyjaszczyk M, Goch JH. Genetic insight into syncopal tilted population with severe clinical presentation. Auton Neurosci 147 (1-2): 97-100.

14. Huang YJ, Bao LM, Wang JY, Huang M. Association between the polymorphism of GNB3C825T gene and vasovagal syncope in children. Zhonghua Er Ke Za Zhi 48 (12): 896-899.

15. Sorrentino S, Forleo C, Iacoviello $M$ et al. Lack of association between genetic polymorphism affecting sympathetic activity and tiltinduced vasovagal syncope. Auton Neurosci 2010; 155: 98-103.

16. Bourne HR. How receptors talk to trimeric G proteins. Curr Opin Cell Biol 1997; 9: 134-142.

17. Sprang SR. G protein mechanisms: insights from structural analysis. Annu Rev Biochem 1997; 66: 639-678.

18. Ansari-Lari MA, Muzny DM, Lu J et al. A gene-rich cluster between the CD4 and triosephosphate isomerase genes at human chromosome 12p13. Genome Res 1996; 6: 314-326.

19. Rosskopf D, Busch S, Manthey I, Siffert W. G protein B3 gene. Structure, promoter, and additional polymorphisms. Hypertension 2000; 36: $33-41$.

20. Siffert W, Rosskopf D, Siffert G et al. Association of a human Gprotein beta3 subunit variant with hypertension. Nat Genet 1998; 18: 45-48.

21. Semplicini A, Siffert W, Sartori M et al. G protein beta3 subunit gene $825 \mathrm{~T}$ allele is associated with increased left ventricular mass in young subjects with mild hypertension. Am J Hypertens 14: 1191-1195.

22. Siffert W, Forster P, Jockel KH et al. Worldwide ethnic distribution of the $\mathrm{G}$ protein beta 3 subunit $825 \mathrm{~T}$ allele and its association with obesity in Caucasian, Chinese, and Black African individuals. J Am Soc Nephrol 1999; 10: 1921-1930.

23. Wascher TC, Paulweber B, Malaimare $L$ et al. Associations of a human $\mathrm{G}$ protein beta3 subunit dimorphism with insulin resistance and carotid atherosclerosis. Stroke 34: 605-609.
24. Luo M, Zheng H, Xu H, Cui B, Wang Z. Association between polymorphism of the G-protein 33 subunit $\mathrm{C} 825 \mathrm{~T}$ and essential hypertension: an updated meta-analysis involving 36,802 subjects. Biol Res 2013; 46: 265-273.

25. Nakao R, Tanaka H, Takitani K et. al. GNB3 C825T polymorphism is associated with postural tachycardia syndrome in children. Pediatrics International 2012 Japan Pediatric Society.

26. Pérez-Paredes $\mathbf{M}$ et al. Results of shortened head-up tilt testing potenciated with sublingual nitroglicerin in a large cohort of patients with unexplained syncope. Eur Heart J 2002; 23 (Suppl): 286.

27. Guzman CE, Sanchez GM, Márquez MF, Hermosillo AG, Cárdenas M. Differences in heart rate variability between cardioinhibitory and vasodepressor responses to head-up tilt table testing. Arch Med Res 1999; 30: 203-211.

28. Gielerak G, Makowski K, Kramarz E et al. Heart rate variability during head-up tilt test in patients with syncope of unknown origin. Kardiol Pol 2002; 57: 399-406.

29. Mehlsen J, Kaijer MN, Mehlsen AB. Autonomic and electrocardiographic changes in cardioinhibitory syncope. Europace 2008; 10: 91-95.

30. Duplyakov D, Golovina G, Sysuenkova E, Garkina S. Can the result of a tilt test be predicted in the first five minutes? Cardiol J 2011; 18: 521-526.

31. Lipsitz LA, Mietus J, Moody GB, Goldberger AL. Spectral characteristics of heart rate variability before and during postural tilt. Relations to aging and risk of syncope. Circulation 1990; 81: 1803-1810.

32. Kochiadakis GE, Kanoupakis EM, Igoumenidis NE et al. Spectral analysis of heart rate variability during tilt-table testing in patients with vasovagal syncope. Int J Cardiol 1998; 64: 185-194.

33. Kouakam C, Lacroix D, Zghal $\mathbf{N}$ et al. Inadequate sympathovagal balance in response to orthostatism in patients with unexplained syncope and a positive headup tilt test. Heart 1999; 82: 312-318.

34. Stewart JM, Erb M, Sorbera C. Heart rate variability and the outcome of head-up tilt in syncopal children. Pediatr Res 1996; 40: 702-709.

35. Evrengul H, Tavli V, Evrengul H, Tavi T, Dursunoglu D. Spectral and timedomain analyses of heart-rate variability during head-upright tilttable testing in children with neurally mediated syncope. Pediatr Cardiol 2006; 27: 670-678.

36. Furlan R, Piazza S, Barbic F et al. Cardiac autonomic patterns preceding occasional vasovagal reactions in healthy humans. Circulation 1998: 1756-1761.

37. Hruboň A, Lacko A, Urban M, Straka J, Bestvina D. Spektrálna analýza variability RR intervalov v diagnostike vazovagálnych synkopálnych stavov. Cardiol 2005; 14 (1): 11-15. 